\title{
Platelet Count Does Not Predict Bleeding in Cirrhotic Patients: Results from the PRO-LIVER Study
}

\author{
S. Basili, MD¹, V. Raparelli, MD, PhD², L. Napoleone, MD², G. Talerico, MD², G.R. Corazza, MD³, F. Perticone, MD4, D. Sacerdoti, MD5,

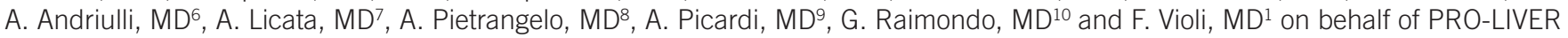 \\ Collaborators ${ }^{11}$
}

OBJECTIVES: Thrombocytopenia is a hallmark for patients with cirrhosis and it is perceived as a risk factor for bleeding events. However, the relationship between platelet count and bleeding is still unclear.

METHODS: $\quad$ We investigated the relationship between platelet count and major or clinical relevant nonmajor bleedings during a follow-up of $\sim 4$ years.

RESULTS: $\quad$ A total of 280 cirrhotic patients with different degrees of liver disease $(67 \%$ males; age $64 \pm 37$ years; $47 \%$ Child-Pugh B and C) were followed up for a median of 1,129 (interquartile range: 800 1,498 ) days yielding 953.12 patient-year of observation. The annual rate of any significant bleeding was 5.45\%/year (3.57\%/year and $1.89 \% / y e a r$ for major and minor bleeding, respectively). Fifty-two (18.6\%) patients experienced a major $(n=34)$ or minor $(n=18)$ bleeding event, predominantly from gastrointestinal origin. Platelet counts progressively decreased with the worsening of liver disease and were similar in patients with or without major or minor bleeding: a platelet count $\leq 50 \times 10^{3} / \mu \mathrm{I}$ was detected in $3(6 \%)$ patients with and in $20(9 \%)$ patients without any bleeding event. Conversely, prothrombin time-international normalized ratio was slightly higher in patients with overall or major bleeding. On Cox proportional hazard analysis, only a previous gastrointestinal bleeding (hazard ratio (HR): 1.96 ; 95\% confidence interval: $1.11-3.47 ; P=0.020$ ) and encephalopathy (HR: $2.05 ; 95 \%$ confidence interval: $1.16-3.62 ; P=0.013$ ) independently predicted overall bleeding events.

CONCLUSIONS: Platelet count does not predict unprovoked major or minor bleeding in cirrhotic patients.

Am J Gastroenterol advance online publication, 19 December 2017; doi:10.1038/ajg.2017.457

\section{INTRODUCTION}

Cirrhosis is associated with laboratory variable changes indicating the presence of a coagulopathy that could theoretically predispose to bleeding. Thus, global clotting tests, such as prothrombin time and activated partial thromboplastin time, are prolonged as a consequence of impaired clotting factor synthesis by liver failure (1). The clinical relevance of this finding has been long questioned as such changes do not reflect in vivo clotting activation that is, in fact, increased more than decreased in cirrhosis (2-4). In particular, we reported an enhanced rate of thrombin generation in cirrhotic patients depending on the degree of liver failure that was more marked in portal compared with systemic circulation (5). This finding leads to suggest that endotoxemia from gut microbiota could exert a procoagulant effect via upregulation of Tissue Factor, a glycoprotein that converts factor X to Xa (6). In accordance with this hypothesis, administration of nonabsorbable antibiotic resulted in coincident lowering of endotoxemia and thrombin generation (7). The paradoxical coexistence of enhanced thrombin generation with activated partial thromboplastin time prolongation may be dependent on the fact that clotting tests lack sensitivity to the concomitant downregulation of anticoagulants that are also poorly synthetized in case of liver

\footnotetext{
${ }^{1}$ Department of Internal Medicine and Medical Specialties, Sapienza University of Rome, Rome, Italy; ${ }^{2}$ Department of Experimental Medicine, Sapienza University of Rome, Rome, Italy; ${ }^{3}$ Clinica Medica I, Department of Internal Medicine S. Matteo, University Hospital Pavia, Pavia, Italy; ${ }^{4}$ Department of Medical and Surgical Sciences, University Magna Græcia of Catanzaro, Catanzaro, Italy; ${ }^{5}$ Department of Medicine, University of Padova, Padova, Italy; ${ }^{6}$ Division of Gastroenterology, Casa Sollievo Sofferenza Hospital, IRCCS, San Giovanni Rotondo, Italy; ${ }^{7}$ Department of Internal Medicine and Specialities, DIBIMIS, University of Palermo,

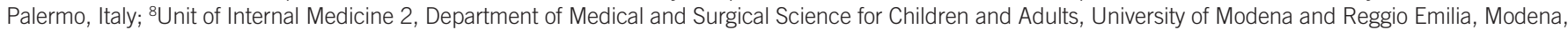
Italy; Internal Medicine and Hepatology Unit, University Campus Bio-Medico, Rome, Italy; ${ }^{10}$ Department of Clinical and Experimental Medicine, University of Messina, Messina, Italy; ${ }^{11}$ The PRO-LIVER Collaborators are listed above the References. Correspondence: F. Violi, MD, Clinica Medica I, Department of Internal Medicine and Medical Specialties, Sapienza University of Rome, Viale del Policlinico 155, Rome 00161, Italy. E-mail: francesco.violi@uniroma1.it Received 20 April 2017; accepted 6 November 2017
} 
failure (8). In accordance with this, there is no correlation between bleeding risk and clotting tests such as prothrombin time and activated partial thromboplastin time in cirrhosis $(2,3)$.

Platelet dysfunction is another putative component of coagulopathy that has been suggested as contributing to bleeding. The presence of coagulopathy in cirrhosis has been based on ex vivo studies demonstrating impaired platelet response to common agonists (9). However, the existence of platelet dysfunction has been recently questioned as the coexistent low platelet count makes it difficult in performing and interpreting ex vivo aggregation test in cirrhosis (9). Conversely, using more adequate laboratory tests, we demonstrated that platelets from cirrhotic patients are hyperresponsive to common agonists with a mechanism involving upregulation of intracellular signaling implicated in platelet activation (10). Low platelet count is another variable that typically occurs in patients with severe liver failure and may predispose to bleeding but its clinical impact is still to be clarified. We have previously reported that bleeding time, which is in part sensitive to platelet count, does not predict bleeding risk in cirrhosis but, because of important influence of vessel vasoconstriction on bleeding time, these data were inadequate (11). Inconclusive data have also been reported when platelet count was taken into account as a laboratory variable to predict bleeding risk (9). Thus, cross-sectional studies provided equivocal data regarding the association between platelet count and spontaneous and provoked bleedings (12-14). To the best of our knowledge, there is only one study that prospectively analyzed the impact of platelet count on bleeding risk in cirrhotic patients. The study, which included essentially patients with platelet count $<150 \times 10^{3} / \mu 1$ and analyzed only the occurrence of variceal bleeding, demonstrated no relationship between platelet count and bleeding risk (15). In order to further explore the role of platelet count on overall bleeding in the "real world" of cirrhotic patients, we prospectively included cirrhotic patients with different degrees of liver disease independently from platelet count. We speculated that if low platelet count actually predisposes to bleeding in cirrhosis, clinical manifestations should not occur exclusively in the gastrointestinal (GI) tract. The goal of the study was, therefore, to assess whether platelet count was predictive of GI or non-GI bleeding or both during a follow-up of $\sim 4$ years.

\section{METHODS}

The PRO-LIVER study is an ongoing Italian-based prospective multicenter study with the primary objective to estimate the prevalence of portal vein thrombosis in a cohort of patients with cirrhosis. This study was conducted in accordance with the EU Note for Guidance on Good Clinical Practice CPMP/ECH/135/95 and the Declaration of Helsinki. Informed consent was obtained from all individual participants included in the study. The study was initiated only after local and ethic approval requirements were obtained (ClinicalTrials.gov Identifier: NCT01470547). The Center participation to the registry was voluntary and not sponsored.

The study reports a prespecified analysis in a subgroup of the entire PRO-LIVER cohort that aimed at exploring the relationship between platelets count and major or clinical relevant nonmajor bleeding defined using the International Society on Thrombosis and Haemostasis (ISTH) criteria.

ISTH major bleeding in nonsurgical patients is defined as having a symptomatic presentation as follows $(16,17)$ : (i) fatal bleeding, and/or (ii) bleeding in a critical area or organ (intracranial, intraspinal, intraocular, retroperitoneal, intra-articular, pericardial, intramuscular with compartment syndrome), and/or (iii) bleeding causing a fall in hemoglobin level of $20 \mathrm{~g} / \mathrm{l}$ or more, or leading to transfusion of two or more units of whole blood or red cells.

Any sign or symptom of hemorrhage that (i) required medical intervention by a healthcare professional or (ii) led to hospitalization or increased level of care or (iii) prompted a face-to-face evaluation had been considered a clinical relevant nonmajor bleeding.

\section{Study population}

All consecutive cirrhotic out- or in-patients who were referred to the 11 centers participating in the substudy were enrolled. The presence of concomitant neoplasms was the only exclusion criteria. Thus, we included patients with a diagnosis of cirrhosis of any etiology and severity. Both Child-Pugh and MELD (Model For End-Stage Liver Disease) scores were assessed to establish the severity of liver disease. Among laboratory variables, prothrombin time-international normalized ratio (PT-INR), total bilirubin, serum albumin, serum creatinine, and platelet counts were mandatory for this substudy.

Data collection and validation have been previously described (18). Briefly, patient identification name was registered in the participating centers, but was not transferred to the central database. Patients have been identified by a serial number for each center. A final database was created and validated by the study coordinator. A phone call interview was scheduled every 3 months to record all relevant clinical events. A follow-up visit was scheduled every 6 months. At each visit all relevant clinical events, the severity of liver disease, and treatments received during the follow-up period were checked.

\section{Statistical analysis}

All continuous variables were tested for normality with the Shapiro-Wilk test. Variables with normal distribution were expressed as mean and s.d., and tested for differences with the Student's $t$-test. Nonnormal variables were expressed as median and interquartile range (IQR) and differences tested with the Mann-Whitney $U$-test. Categorical variables were expressed as counts and percentages and were analyzed by the $\chi^{2}$ test.

Survival curves were formally compared using the log-rank test. Cox proportional hazards analysis was used to calculate the adjusted relative hazards of outcome events by each clinical variable.

Stochastic level of entry into the model was set at a $P$ value $=0.10$, and interaction terms were explored for all the variables in the final model.

Only $P$ values $<0.05$ were regarded as statistically significant. All tests were two-tailed and analyses were performed using computer software packages (SPSS-22.0, IBM, Armonk, NY). 


\section{RESULTS}

Overall, 280 cirrhotic patients were recruited (188 males; 92 females; age $64 \pm 37$ years). Viral $(n=122,44 \%)$, alcoholic $(n=79$, $28 \%)$, and both viral and alcoholic $(n=38,14 \%)$ etiologies were predominant. Compensated cirrhotic patients (Child-Pugh score: A class) were 53 and $47 \%$ cirrhotic patients had moderate-severe disease (87 of B and 45 of C class). Mean MELD score was $11 \pm 6$. Ascites was detected in 120 (43\%) patients: 77 (64\%) responsive to diuretic therapy and $43(36 \%)$ had a refractory ascites. At the time of enrollment, hepatic encephalopathy (HE) affected 62 (22\%) patients (53 were in I-II and 9 in III-IV grades). Sixtyfive $(23 \%)$ patients had previous GI bleeding. Compared with the entire cohort, these patients had similar MELD score (11.5 \pm 5.4 vs. $11.2 \pm 5.7$ ), percentage of moderate-severe disease ( $52 \%$ vs. $45 \%$, $P=0.34$ ), ascites (58\% vs. $57 \%$ ), and HE (26\% vs. $21 \%)$. Moreover, among 19 patients who experienced GI bleeding in the previous 12 months, $~ 50 \%$ had HE at the time of enrollment. Recent esophagogastroduodenoscopy has been reported in 229 out of 280 patients. Among them, 90 (30\%) had no esophageal varices. The remaining 139 patients had esophageal varices (86 were classified as small (F1), 44 as medium (F2), and 9 as large (F3)) (19).

Sixteen patients $(6.7 \%)$ were on anticoagulants (14 received lowmolecular-weight heparin and 2 vitamin-K antagonist). None of the patients with previous GI bleeding was treated with any kind of anticoagulants.

In all, 145 (52\%) patients received aldosterone receptor antagonist, 139 (50\%) loop diuretics, and 104 (37\%) $\beta$-blockers. Thirteen (4.6\%) patients were previously treated with endoscopic variceal ligation and 2 with esophageal variceal sclerosis.

Mean serum levels of creatinine and albumin were $0.93 \pm 0.47$ and $3.39 \pm 0.61 \mathrm{~g} / \mathrm{l}$, respectively. Median bilirubin was 1.16 (IQR: $0.80-2.0) \mathrm{mg} / \mathrm{dl}$ and mean PT-INR was $1.3 \pm 0.3$ (median (IQR): $1.2(1.1-1.4)$ ). Platelet counts were $120 \pm 63 \times 10^{3} / \mu$ l. Platelet counts $\leq 50 \times 10^{3} / \mu$ l were detected in $23(8 \%)$ patients, between 50 and $150 \times 10^{3} / \mu \mathrm{l}$ in $184(66 \%)$ patients, and $\geq 150 \times 10^{3} / \mu \mathrm{l}$ in $73(26 \%)$ patients. Platelet counts progressively decreased with worsening of liver disease $\left(129 \pm 67 \times 10^{3} / \mu \mathrm{l}\right.$ in Child-Pugh A class, $114 \pm 58 \times 10^{3} / \mu \mathrm{l}$ in $\mathrm{B}$ class, and $\left.105 \pm 57 \times 10^{3} / \mu \mathrm{l}, P=0.0464\right)$. An inverse significant correlation between platelet counts, ChildPugh score ( $\mathrm{r}=-0.13, P=0.030)$, MELD score $(\mathrm{r}=-0.17, P=0.006)$, and PT-INR ( $r=-0.30, P<0.0001)$ was also observed; conversely, albumin serum levels significantly correlated with platelet counts $(\mathrm{r}=0.15, P=0.010)$.

During the observational period (median length of follow-up was 1,129 (IQR: 800-1,498) days yielding 953.12 patient-years of observation) $52(18.6 \%)$ patients experienced a major or minor bleeding event. Thus, the annual rate of any significant bleeding was $5.45 \%$ /year.

\section{Major bleeding events}

Thirty-four patients (12\%) met the criteria for major events; of them, 31 (91\%) experienced a bleeding related to portal hypertension (bleeding episode secondary to the rupture or erosion of esophageal or gastric varices and/or portal hypertensive gastropathy, manifested clinically as melena or hematemesis) that was fatal in 5 patients. At the enrollment, 19 (61\%) had gastroesophageal varices $(F 1=12, F 2=4, F 3=3)$ : only 8 of them received $\beta$-blockers or/and endoscopic variceal ligation/sclerosis. Conversely, seven patients without gastroesophageal varices received prophylaxis with $\beta$-blockers.

The remaining three included two intracerebral hemorrhage (fatal in one patient) and one from bronchial tree; the median follow-up for the index event was 195 (IQR: 90-553) days. Thus, the annual rate of major bleeding was 3.57\%/year. Patients with major bleeding events during the follow-up did not differ in terms of age, sex, Child-Pugh score, etiology, diuretic therapy, and $\beta$-blockers (44\% vs. 36\%) with those without major bleedings.

MELD score was higher in patients who experienced major bleeding during the follow-up ( $13.3 \pm 5.7$ vs. $10.9 \pm 5.5, P=0.0215)$. A higher percentage of previous gastrointestinal bleeding characterized patients with major bleeding during the follow-up ( $41 \%$ vs. $21 \%, P=0.008$ ).

Patients with major bleeding had more frequently encephalopathy ( $41 \%$ vs. $19.5 \%, P=0.017$ ); ascites was present in $47 \%$ of patients with and in $42 \%$ of patients without major bleeding.

Serum albumin, creatinine, and bilirubin median levels were similar between patients with and without major bleeding events.

Median PT-INR ((IQR): $1.4(1.1-1.5)$ vs. $1.2(1.1-1.35) P=0.042)$ was slightly higher in patients with major bleeding. Conversely, platelet counts were similar in patients with and without major bleeding $\left(114 \pm 56\right.$ vs. $\left.121 \pm 64 \times 10^{3} / \mu \mathrm{l}\right)$. Among patients who experienced major bleeding, $3(9 \%)$ had platelet counts $\leq 50 \times 10^{3} / \mu 1,20$ (59\%) between 50 and $150 \times 10^{3} / \mu \mathrm{l}$, and $11(32 \%)>150 \times 10^{3} / \mu \mathrm{l}$.

The multivariable Cox regression analysis was performed including variables with a $P$ value $=0.10$ at the univariate analysis (Tables 1 and 2, model A). The final model of multivariable Cox regression analysis showed that presence of $\mathrm{HE}$ (hazard ratio (HR): $2.41 ; P=0.012$ ) (Figure 1a) and previous GI bleeding (HR: 2.29; $P=0.018$ ) (Figure $1 \mathbf{b}$ ) independently predicted major bleeding events, after adjusting for MELD score and $\beta$-blockers treatment. In addition, to ensure that one parameter of MELD score (e.g., INR, creatinine, bilirubin) did not dominate the analysis, the proportional hazards model was run using each component of MELD individually (Table 2, model B). The final model confirmed that HE (HR: 2.47; $P=0.010$ ) and previous GI bleeding (HR: 2.40; $P=0.012$ ) independently predicted major bleeding events.

\section{Minor bleeding events}

The median follow-up of 18 patients who had minor bleeding events was 186 (IQR: 30-658) days. Thus, the annual rate of minor bleedings was $1.89 \%$ /year. All but one with ecchymosis had bleedings from gastrointestinal tube (upper gastrointestinal system $(n=10)$, cecum or ascending colon $(n=4)$, rectal bleeding $(n=3))$.

\section{Patient characteristics associated to all bleeding events}

Clinical and laboratory characteristics of patients according to the presence of major or nonmajor bleeding are depicted in Table 3.

No differences in age, sex, and etiology were observed between the two groups. Patients with bleedings had more advanced 


\begin{tabular}{|c|c|c|c|}
\hline & Hazard ratio & $95 \% \mathrm{Cl}$ & $P$ \\
\hline Age (per year) & 0.99 & $0.96-1.02$ & 0.571 \\
\hline Male sex & 0.99 & $0.48-2.01$ & 0.986 \\
\hline Ascites & 1.23 & $0.63-2.42$ & 0.540 \\
\hline Encephalopathy & 2.69 & $1.36-5.34$ & 0.004 \\
\hline Previous GI bleeding & 2.56 & $1.29-5.06$ & 0.007 \\
\hline \multicolumn{4}{|l|}{$C-P$ classes } \\
\hline Class C-P-B vs. C-P-A & 1.41 & $0.64-3.10$ & 0.396 \\
\hline Class C-P-C vs. C-P-A & 2.35 & $1.02-5.44$ & 0.045 \\
\hline Esophageal varices $\geq F 2$ & 0.91 & $0.39-2.10$ & 0.828 \\
\hline Esophageal varices $\geq F 1$ & 0.93 & $0.46-1.88$ & 0.842 \\
\hline MELD score & 1.05 & $1.00-1.10$ & 0.019 \\
\hline Platelet count $\left(\times 10^{3} / \mu \mathrm{l}\right)$ & 0.998 & $0.993-1.00$ & 0.572 \\
\hline Platelet count $\left(<50 \times 10^{3} / \mu \mid\right)$ & 1.03 & $0.31-3.36$ & 0.965 \\
\hline PT-INR & 2.27 & $1.04-4.92$ & 0.038 \\
\hline Albumin (g/dl) & 0.63 & $0.36-1.08$ & 0.093 \\
\hline Bilirubin (mg/dl) & 0.99 & $0.89-1.09$ & 0.790 \\
\hline Creatinine (mg/dl) & 1.24 & $0.73-2.12$ & 0.421 \\
\hline$\beta$-Blocker treatment & 0.71 & $0.36-1.4$ & 0.319 \\
\hline
\end{tabular}

Table 2. Multivariate analyses according to the incidence of major bleeding during the observation period

\begin{tabular}{llllc} 
& HR & \multicolumn{2}{c}{$95 \%$ Cl } & $P$ \\
Model A & & & & \\
\hline Encephalopathy & 2.411 & 1.216 & 4.781 & 0.012 \\
Previous Gl bleeding & 2.288 & 1.154 & 4.536 & 0.018 \\
MELD score & 1.033 & 0.973 & 1.097 & 0.286 \\
Albumin (g/dl) & 0.974 & 0.502 & 1.887 & 0.937 \\
Model B & & & & \\
Encephalopathy & 2.475 & 1.248 & 4.912 & 0.010 \\
Previous Gl bleeding & 2.403 & 1.211 & 4.766 & 0.012 \\
PT-INR & 2.060 & 0.723 & 5.871 & 0.176 \\
Creatinine (mg/dl) & 1.155 & 0.613 & 2.177 & 0.655 \\
Bilirubin (mg/dl) & 0.892 & 0.761 & 1.046 & 0.160 \\
Albumin (g/dl) & 0.777 & 0.398 & 1.516 & 0.459
\end{tabular}

$\mathrm{Cl}$, confidence interval; Gl, gastrointestinal; HR, hazard ratio; MELD, Model For End-Stage Liver Disease; PT-INR, prothrombin time-international normalized ratio.

Model A: proportional hazards model was run using variables showed $P$ value $=0.10$ at the univariate analysis.

Model B: proportional hazards model was run using each component of MELD individually.
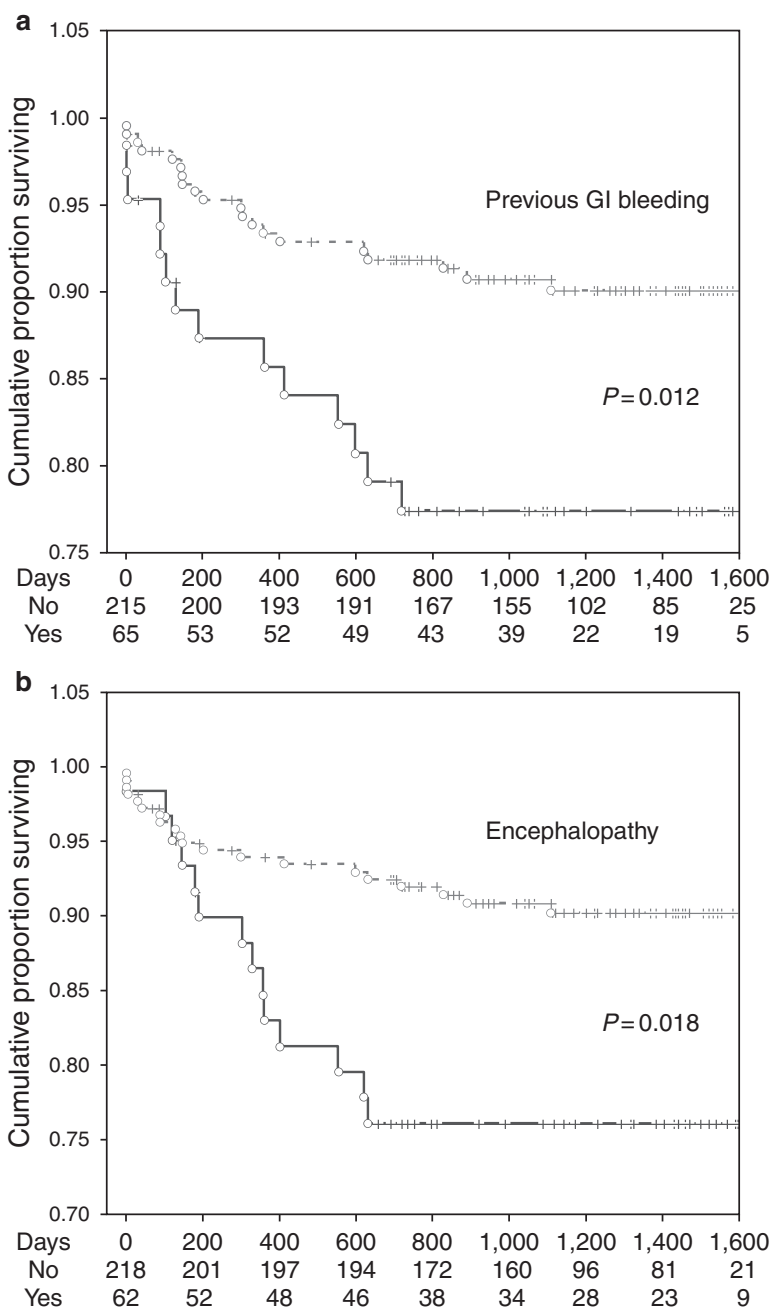

Figure 1. Kaplan-Meier curves for major bleeding in PRO-LIVER cohort. Kaplan-Meier estimates of time to major bleeding according to (a) previous gastrointestinal (GI) bleeding and (b) encephalopathy. $\mathrm{No}=$ patients without previous gastrointestinal bleeding or encephalopathy; Yes=patients with previous gastrointestinal bleeding or encephalopathy.

disease, higher percentage of previous gastrointestinal bleeding, and more frequently encephalopathy. Esophageal varices of grade $\geq 2$, ascites, serum albumin, creatinine, and bilirubin median levels did not discriminate the two groups.

Median PT-INR was slightly higher in patients with all types of bleeding. Otherwise, platelet counts did not discriminate patients with and without bleedings. In particular, platelet counts $\leq 50 \times 10^{3} / \mu \mathrm{l}$ were detected in $3(6 \%)$ patients with and in $20(9 \%)$ without any bleeding event.

Table 4 reported variables univariate analysis and variables that entered the multivariate multivariable Cox regression analysis $(P=0.10)$ (Table 5, model A). The final model showed that only previous GI bleeding (HR: 1.96; $P=0.020$ ) (Figure 2a) and encephalopathy (HR: 2.05; $P=0.013$ ) (Figure $2 \mathbf{b}$ ) were significantly associated with bleeding during the follow-up after adjusting for serum albumin, $\beta$-blocker treatment, and MELD score. Using each component of MELD individually (Table 5, model B), the final model 
Table 3. Clinical characteristics of patients according to all bleeding events (major or minor) experienced during the follow-up

\begin{tabular}{|c|c|c|c|}
\hline Variables & $\begin{array}{l}\text { Bleedings, } \\
\quad N=52\end{array}$ & $\begin{array}{c}\text { No bleedings, } \\
N=228\end{array}$ & $P$ \\
\hline Age (years) & $64.7 \pm 11.2$ & $63.9 \pm 11.7$ & 0.6564 \\
\hline Males, $n(\%)$ & $36(69)$ & $152(67)$ & 0.7224 \\
\hline Etiology, n (\%) & & & 0.2557 \\
\hline Alcohol & $18(35)$ & $61(27)$ & \\
\hline Others & $34(65)$ & $167(73)$ & \\
\hline Child-Pugh score & & & 0.0615 \\
\hline Class A, $n(\%)$ & $20(38)$ & $128(56)$ & \\
\hline Class B, $n(\%)$ & $20(38)$ & $67(29)$ & \\
\hline Class C, $n(\%)$ & $12(24)$ & $33(15)$ & \\
\hline $\begin{array}{l}\text { Child-Pugh score } \\
\text { median (IQR) }\end{array}$ & $7(6-9)$ & $6(5-8)$ & 0.0461 \\
\hline $\begin{array}{l}\text { MELD score median } \\
\text { (IQR) }\end{array}$ & $12(9-14)$ & $9(7-12)$ & 0.0193 \\
\hline Esophageal varices & & & 0.6232 \\
\hline $\mathrm{F} 1, n(\%)$ & $18(37)$ & $68(38)$ & \\
\hline $\mathrm{F} 2, n(\%)$ & 7 (14) & $37(21)$ & \\
\hline $\mathrm{F} 3, n(\%)$ & $3(6)$ & $6(3)$ & \\
\hline Ascites, $n(\%)$ & 27 (51.9) & $93(40.8)$ & 0.143 \\
\hline $\begin{array}{l}\text { Encephalopathy, } \\
n(\%)\end{array}$ & $20(38.5)$ & $42(18.4)$ & 0.0071 \\
\hline $\begin{array}{l}\text { Previous GI bleeding, } \\
n(\%)\end{array}$ & $19(36.5)$ & $46(20.2)$ & 0.0117 \\
\hline $\begin{array}{l}\text { Platelet counts } \\
\left(\times 10^{\circ} / 1\right)\end{array}$ & $117 \pm 59$ & $121 \pm 64$ & 0.7552 \\
\hline $\begin{array}{l}\text { Platelet counts } \\
\leq 50 \times 10^{9} /, n(\%)\end{array}$ & $3(6)$ & $20(9)$ & 0.7209 \\
\hline $\begin{array}{l}\text { Platelet counts }>50 \\
\text { and }<150 \times 10^{9} / / \\
n(\%)\end{array}$ & $34(65)$ & $150(66)$ & \\
\hline $\begin{array}{l}\text { Platelet counts } \\
\geq 150 \times 10 \% /, n(\%)\end{array}$ & $15(29)$ & $58(25)$ & \\
\hline PT-INR & $1.30(1.12-1.48)$ & $1.20(1.06-1.33)$ & 0.0029 \\
\hline Creatinine (mg/dl) & $0.98 \pm 0.42$ & $0.92 \pm 0.48$ & 0.4050 \\
\hline Albumin $(g / l)$ & $3.23 \pm 0.50$ & $3.42 \pm 0.63$ & 0.0373 \\
\hline Bilirubin (mg/dl) & $2.02 \pm 2.17$ & $2.28 \pm 4.15$ & 0.6644 \\
\hline $\begin{array}{l}\text { Aldosterone receptor } \\
\text { antagonist, } n(\%)\end{array}$ & $31(60)$ & $114(50)$ & 0.2105 \\
\hline Loop diuretics, $n(\%)$ & $31(60)$ & $108(47)$ & 0.1109 \\
\hline$\beta$-Blockers, $n(\%)$ & $25(48)$ & $79(35)$ & 0.0705 \\
\hline
\end{tabular}

GI, gastrointestinal; IQR, interquartile range; MELD, Model For End-Stage Liver Disease; PT-INR, prothrombin time-international normalized ratio.

showed that encephalopathy (HR: 2.13; $P=0.015$ ), previous GI bleeding (HR: 1.87; $P=0.031$ ), and PT-INR (HR: 2.65; $P=0.012$ ) independently predicted all bleeding events.
Table 4. Univariate analyses according to the incidence of all bleeding (major and minor) during the observational period

$\begin{array}{lccc} & \text { Hazard ratio } & 95 \% \text { Cl } & P \\ \text { Age (per year) } & 0.66 & 0.98-1.02 & 0.663 \\ \text { Male sex } & 1.08 & 0.60-1.95 & 0.794 \\ \text { Ascites } & 1.49 & 0.86-2.57 & 0.149 \\ \text { Encephalopathy } & 2.40 & 1.37-4.19 & 0.002 \\ \text { Previous Gl bleeding } & 2.10 & 1.20-3.70 & 0.010 \\ \text { C-P classes } & & & \\ \quad \text { Class C-P-B vs. C-P-A } & 1.78 & 0.96-3.31 & 0.068 \\ \quad \text { Class C-P-C vs. C-P-A } & 2.18 & 1.07-4.47 & 0.033 \\ \text { Esophageal varices } \geq F 2 & 0.83 & 0.41-1.66 & 0.596 \\ \text { Esophageal varices } \geq F 1 & 0.84 & 0.48-1.48 & 0.551 \\ \text { MELD score } & 1.04 & 1.00-1.08 & 0.019 \\ \text { Platelet count }\left(\times 10^{3} / \mu \mathrm{l}\right) & 0.999 & 0.995-1.004 & 0.764 \\ \text { Platelet count }\left(<50 \times 10^{3} / \mu l\right) & 0.65 & 0.20-2.08 & 0.467 \\ \text { PT-INR } & 2.31 & 1.24-4.29 & 0.008 \\ \text { Albumin (g/dl) } & 0.63 & 0.40-0.98 & 0.042 \\ \text { Bilirubin (mg/dl) } & 0.98 & 0.91-1.06 & 0.790 \\ \text { Creatinine (mg/dl) } & 1.22 & 0.78-1.90 & 0.373 \\ \text { B-Blocker treatment } & 0.60 & 0.35-1.04 & 0.068\end{array}$

$\mathrm{Cl}$, confidence interval; CP, Child-Pugh; GI, gastrointestinal; MELD, Model For End-Stage Liver Disease; PT-INR, prothrombin time-international normalized ratio.

\section{DISCUSSION}

The results of this prospective study in cirrhotic patients with different degrees of liver failure show that bleeding complication occurs prevalently from gastrointestinal tube and that platelet count is not predictive of overall bleeding risk.

In our cohort, the annual rate of overall bleeding was 5.45\%/ year, in accordance with previous data mostly considering variceal hemorrhage as a major outcome; in fact, variceal bleeding occurs at a yearly rate between 5 and 15\%, depending on cirrhosis severity and variceal size (20). Data on other types of bleeding, mainly derived by cross-sectional and retrospective studies, do not permit adequate comparison (21).

In our cohort, $>90 \%$ of major or minor bleedings recognized a gastrointestinal source; major upper GI bleedings from esophageal varices were observed in $~ 55 \%$ of cases. Similar to previous studies (22-24), the remaining GI hemorrhagic events recognized a nonvariceal upper GI source or lower GI origin.

Of the remaining bleeding complications, 4 (7.7\%) were not from gastrointestinal tube but one cutaneous ecchymosis, one hemoptysis, and two intracerebral hemorrhages, indicating that in cirrhosis bleeding is essentially gastrointestinal. Thus, in accordance with previous reports showing that intracerebral hemorrhage is a very rare complication $(25,26)$, we found this complication in two patients of the entire cohort corresponding to $0.17 \% /$ year. 


\begin{tabular}{|c|c|c|c|c|}
\hline & HR & \multicolumn{2}{|c|}{$95 \% \mathrm{Cl}$} & $P$ \\
\hline \multicolumn{5}{|l|}{ Model A } \\
\hline Encephalopathy & 2.049 & 1.160 & 3.619 & 0.013 \\
\hline Previous GI bleeding & 1.963 & 1.112 & 3.468 & 0.020 \\
\hline MELD score & 1.026 & 0.976 & 1.078 & 0.312 \\
\hline$\beta$-Blocker treatment & 1.533 & 0.882 & 2.664 & 0.130 \\
\hline Albumin (g/dl) & 0.927 & 0.544 & 1.578 & 0.779 \\
\hline \multicolumn{5}{|l|}{ Model B } \\
\hline Encephalopathy & 2.127 & 1.156 & 3.912 & 0.015 \\
\hline Previous GI bleeding & 1.874 & 1.059 & 3.318 & 0.031 \\
\hline PT-INR & 2.656 & 1.236 & 5.704 & 0.012 \\
\hline Bilirubin (mg/dl) & 0.892 & 0.783 & 1.017 & 0.088 \\
\hline Creatinine (mg/dl) & 1.074 & 0.632 & 1.827 & 0.791 \\
\hline Albumin (g/dl) & 0.798 & 0.469 & 1.359 & 0.407 \\
\hline$\beta$-Blocker treatment & 1.540 & 0.885 & 2.679 & 0.126 \\
\hline \multicolumn{5}{|c|}{$\begin{array}{l}\mathrm{Cl} \text {, confidence interval; GI, gastrointestinal; HR, hazard ratio; MELD, Model For } \\
\text { End-Stage Liver Disease; PT-INR, prothrombin time-international normalized } \\
\text { ratio. }\end{array}$} \\
\hline \multicolumn{5}{|c|}{$\begin{array}{l}\text { Model A: proportional hazards model was run using variables showed } P \\
\text { value }=0.10 \text { at the univariate analysis. }\end{array}$} \\
\hline \multicolumn{5}{|c|}{$\begin{array}{l}\text { Model B: proportional hazards model was run using each component of MELD } \\
\text { individually. }\end{array}$} \\
\hline
\end{tabular}

Among the clinical variables considered, previous GI bleeding and encephalopathy were associated with an enhanced risk of overall bleedings. Conversely, global clotting tests such as PT were not predictive of major bleedings, in accordance with the previously reported poor predictive value of clotting tests vs. bleeding risk in cirrhosis $(3,27)$. However, we cannot exclude that other clotting tests may be associated with bleedings as evidenced by previous reports where von Willebrand factor, factor VIII/protein $\mathrm{C}$ ratio, and fibrinogen were associated with variceal and nonvariceal bleeding $(12,15,28,29)$.

Our findings reinforce and extend the results of a previous study (15) that demonstrated absence of relationship between platelet count and variceal bleeding in a follow-up of $\sim 3$ years. In particular, we observed that platelet count does not predict bleeding events in cirrhotic patients even considering bleeding type and site. Moreover, the entity of thrombocytopenia seemed to have no impact on bleeding events as patients did not display a higher risk of major or minor bleedings even with a low platelet count $\left(\leq 50 \times 10^{3} / \mu \mathrm{l}\right)$. Taken together, these data lead to suggest that, in cirrhosis, platelets maintain a hemostatically adequate function and this is also consistent with the previously reported lack of association between platelet count and bleeding after invasive procedures (14). It is possible, however, that in particular clinical settings, such as critically ill cirrhotic patients admitted to intensive care units, very low
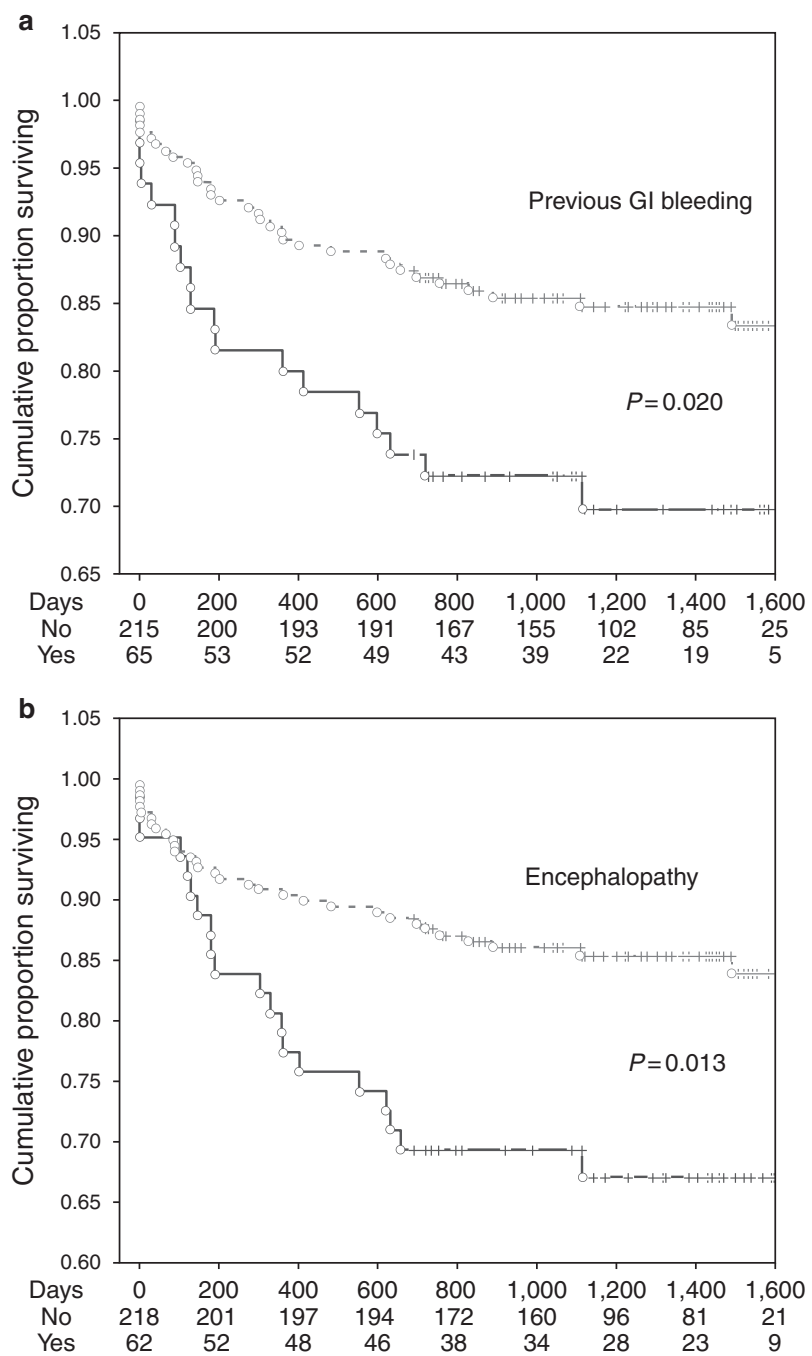

Figure 2. Kaplan-Meier curves for major and minor bleeding in PRO-LIVER cohort. Kaplan-Meier estimates of time to bleedings (minor and major) according to (a) previous gastrointestinal (GI) bleeding and (b) encephalopathy. $\mathrm{No}=$ patients without previous gastrointestinal bleeding or encephalopathy; Yes=patients with previous gastrointestinal bleeding or encephalopathy.

platelet count $\left(<30 \times 10^{3} / \mu \mathrm{l}\right)$ is associated with major bleeding in a context of clinical complexity including sepsis and neurologic disorder (12).

\section{Study limitations}

As platelet counts $<50 \times 10^{3} / \mu 1$ were relatively rare $(8.2 \%)$, this might account for the lack of association between severe thrombocytopenia and bleeding event rate. Furthermore, given the relatively low bleeding rate, we cannot exclude that our sample is underpowered to assess the predictive value of platelet count.

Only $37 \%$ of the cohort with esophageal varices were taking $\beta$-blockers. The increasing controversy about the use of $\beta$-blockers in patients with advanced cirrhosis and complications $(30,31)$ and the design of this study, which did not require a standardize 
therapy for patients with esophageal varices, may explain this low prescription.

\section{Pathophysiological and clinical implications of the study}

Previous studies have suggested that cirrhotic patients suffer not only from thrombocytopenia but also from thrombocytopathy because of impaired platelet response to common agonists (9). Our report questions the existence of platelet-related coagulopathy as the bleeding scenario is not compatible with an impaired platelet function. For example, in case of platelet genetic dysfunction and drug-related platelet inhibition, patients suffer from bleeding such as diffuse ecchymosis or enhanced risk of cerebral hemorrhage $(32,33)$. Furthermore, no increased bleeding was observed in patients with platelet count $\leq 50 \times 10^{3} / \mu 1$, which is apparently in contrast with the fact that in other hematological disorders this platelet count may be associated with enhanced bleeding risk (34). A potential explanation for this finding is that platelets from cirrhosis are actually overactivated with a mechanism related to systemic inflammation and oxidative stress (10) that could, therefore, protect from bleeding even in the case of low platelet count.

An implication of the study regards the need, if any, to increase platelet count in case of elective or urgent surgery. We have previously questioned the need for the use of eltrombopag in cirrhotic patients with low platelet count as it may expand the number of activated platelets so favoring thrombotic complications (35). This issue should be seriously considered in the future.

In conclusion, this study shows that in cirrhosis platelet count is not predictive of bleedings, and hence questioning the existence of a thrombocytopathy and suggesting, conversely, that platelet function is adequately preserved.

\section{PRO-LIVER Study Collaborators}

Palasciano Giuseppe, D’Alitto Felicia, Palmieri Vincenzo Ostilio, Santovito Daniela, Di Michele Dario, Croce Giuseppe, Brocco Silvia, Fasolato Silvano, Cecchetto Lara, Bombonato Giancarlo, Bertoni Michele, Restuccia Tea, Andreozzi Paola, Liguori Maria Livia, Caroleo Benedetto, Perticone Maria, Staltari Orietta, Manfredini Roberto, De Giorgi Alfredo, Averna Maurizio, Giammanco Antonina, Granito Alessandro, Pettinari Irene, Marinelli Sara, Bolondi Luigi, Falsetti Lorenzo, Salvi Aldo, Durante-Mangoni Emanuele, Cesaro Flavio, Farinaro Vincenza, Ragone Enrico, Morana Ignazio, Ippolito Antonio, Iacobellis Angelo, Niro Grazia, Merla Antonio, Maimone Sergio, Cacciola Irene, Varvara Doriana, Drenaggi Davide, Staffolani Silvia,Vespasiani-Gentilucci Umberto, Galati Giovanni, Gallo Paolo, Davì Giovanni, Schiavone Cosima, Santilli Francesca, Tana Claudio, Soresi Maurizio, Bianchi Giovanni Battista, Carderi Isabella, Pinto Antonio, Tuttolomondo Antonino, Ferrari Giovanni, Gresele Paolo, Fierro Tiziana, Morelli Olivia, Laffi Giacomo, Romanelli Roberto Giulio, Arena Umberto, Stasi Cristina, Gasbarrini Antonio, Garcovich Matteo, Zocco Maria Assunta, Riccardi Laura, Ainora Maria Elena, Capeci William, Martino Giuseppe Pio, Nobili Lorenzo, Cavallo Maurizio, Fru- giuele Pierluigi, Greco Antonio, Ventura Paolo, Cuoghi Chiara, Marcacci Matteo, Serviddio Gaetano, Vendemiale Gianluigi, Villani Rosanna, Gargano Ruggiero, Vidili Gianpaolo, Di Cesare Valentina, Masala Maristella, Delitala Giuseppe, Invernizzi Pietro, Vincenzo Ronca, Di Minno Giovanni, Tufano Antonella, Purrello Francesco, Privitera Graziella, Forgione Alessandra, Curigliano Valentina, Senzolo Marco, Rodríguez-Castro Kryssia Isabel, Giannelli Gianluigi, Serra Carla, Neri Sergio, Pignataro Pietro, Rizzetto Mario, Debernardi Venon Wilma, Svegliati Baroni Gianluca, Bergamaschi Gaetano, Masotti Michela, Costanzo Filippo, Antonio Figliomeni, Angelico Francesco, Del Ben Maria, Polimeni Licia, Proietti Marco, Cangemi Roberto, Romiti Giulio Francesco, Toriello Filippo, Sperduti Nicolò, Santangelo Giuseppe, Visioli Giacomo, Todisco Tommaso, Vestri Anna Rita, Farcomeni Alessio, Corrao Salvatore, Gobbi Paolo, Corradini Elena, Costantino Giorgio, Tripepi Giovanni, Angelico Mario, Bolondi Luigi, Granito Alessandro, D’Amico Gennaro, de Franchis Roberto, Gatta Angelo, Tassone Eliezer Joseph, Anzaldi Massimiliano, Barone Milena, Bazzini Cristina, Bianchi Paola Ilaria, Boari Benedetta, Bracco Christian, Buonauro Agostino, Buttà Carmelo, Buzzetti Elena, Calabria Stefano, Caradio Federica, Carleo Pietro, Carrabba Maria Domenica, Castorani Luigi, Cecchetto Lara, Cicco Sebastiano, Cimini Claudia, Colombo Barbara Maria, De Giorgi Alfredo, De Vuono Stefano, Denegri Andrea, Del Corso Lisette, Di Giosia Paolo, Donnarumma Emilia, Giorgini Paolo, Grassi Davide, Grembiale Alessandro, Hijazi Daniel, Iamele Luigi, Lorusso Giusi, Marchese Alessandra, Marra Alberto Maria, Masala Maristella, Miceli Giuseppe, Montebianco Abenavoli Ludovico, Murgia Giuseppe, Naccarato Paola, Padula Donatella, Pattoneri Paolo, Perego Francesca, Pesce Paola, Petramala Luigi, Piano Salvatore, Pinto Daniela, Pinna Miriam, Pignataro Francesca Serena, Pretti Vincenzo, Pucci Giacomo, Salinaro Francesco, Salzano Andrea, Santarossa Claudia, Scarpini Francesca, Scicali Roberto, Sirico Domenico, Suppressa Patrizia, Talia Michela, Torres Daniele, Traversa Matteo, Vazzana Natale, Vecchio Claudia Rita, Vettore Elia, Vitale Francesco.

\section{CONFLICT OF INTEREST}

Guarantor of the article: F. Violi, MD.

Specific author contributions: S. Basili: study design, patient recruitment, data analysis and interpretation, and writing the manuscript. V. Raparelli: data collection, patient recruitment, data analysis and interpretation, and writing the manuscript. L. Napoleone: patient recruitment and data entry. G. Talerico: patient recruitment and data entry. G.R. Corazza: patient recruitment and revision of the manuscript. F. Perticone, D. Sacerdoti, A. Andriulli, A. Licata, A. Pietrangelo, A. Picardi, and G. Raimondo: patient recruitment and revision of the manuscript. F. Violi: study conception and design, data interpretation, and writing the manuscript. F. Violi had full access to all the data in the study and had final responsibility for the decision to submit for publication.

Financial support: SAPIENZA University Research Project 2016. Potential competing interests: None. 


\section{Study Highlights}

\section{WHAT IS CURRENT KNOWLEDGE}

Cirrhosis is associated with thrombocytopenia and, apparently, thrombocytopathy but its clinical impact in the real world is still unclear.

Platelet count $<150 \times 10^{3} / \mu$ l was not associated with variceal bleeding.

Nevertheless, there are no data regarding nonvariceal bleeding.

\section{WHAT IS NEW HERE}

During a follow-up of $\sim 4$ years in cirrhotic patients, major and minor bleedings occurred prevalently $(>90 \%)$ in the gastrointestinal tract.

No relationship between platelet count and overall or gastrointestinal bleedings was observed even considering patients with low platelet count $\left(\leq 50 \times 10^{3} / \mu \mathrm{l}\right)$.

Cirrhosis is complicated essentially by bleeding complication from gastrointestinal tract, whereas nonvariceal bleeding is scarce.

Clinical usefulness of increasing platelet count in cirrhotic patients should be carefully reconsidered.

\section{REFERENCES}

1. Caldwell SH, Hoffman M, Lisman T et al. Coagulation disorders and hemostasis in liver disease pathophysiology and critical assessment of current management. Hepatology 2006;44:1039-46.

2. Violi F. Should the term coagulopathy in cirrhosis be abandoned? JAMA Intern Med 2015;175:862-3.

3. Violi F, Ferro D. Clotting activation and hyperfibrinolysis in cirrhosis: implication for bleeding and thrombosis. Semin Thromb Hemost 2013;39: $426-33$.

4. Ferro D, Angelico F, Caldwell SH et al. Bleeding and thrombosis in cirrhotic patients: what really matters? Dig Liver Dis 2012;44:275-9.

5. Violi F, Ferro D, Basili S et al. Ongoing prothrombotic state in the portal circulation of cirrhotic patients. Thromb Haemost 1997;77:44-7.

6. Saliola M, Lorenzet R, Ferro D et al. Enhanced expression of monocyte tissue factor in patients with liver cirrhosis. Gut 1998;43:428-32.

7. Violi F, Ferro D, Basili S et al. Association between low-grade disseminated intravascular coagulation and endotoxemia in patients with liver cirrhosis. Gastroenterology 1995;109:531-9.

8. Tripodi A, Primignani M, Chantarangkul V et al. An imbalance of pro- vs anti-coagulation factors in plasma from patients with cirrhosis. Gastroenterology 2009;137:2105-11.

9. Violi F, Basili S, Raparelli V et al. Patients with liver cirrhosis suffer from primary haemostatic defects. Fact or fiction? J Hepatol 2011;55:1415-27.

10. Raparelli V, Basili S, Carnevale R et al. Low-grade endotoxemia and platelet activation in cirrhosis. Hepatology 2017;65:571-81.

11. Basili S, Ferro D, Leo R et al. Bleeding time does not predict gastrointestinal bleeding in patients with cirrhosis. The CALC Group. Coagulation Abnormalities in Liver Cirrhosis. J Hepatol 1996;24:574-80.

12. Drolz A, Horvatits T, Roedl K et al. Coagulation parameters and major bleeding in critically ill patients with cirrhosis. Hepatology 2016;64:556-68.

13. Li J, Qi X, Deng H et al. Association of conventional haemostasis and coagulation tests with the risk of acute upper gastrointestinal bleeding in liver cirrhosis: a retrospective study. Gastroenterol Rep (Oxf) 2016;4: 315-9.

14. Napolitano G, Iacobellis A, Merla A et al. Bleeding after invasive procedures is rare and unpredicted by platelet counts in cirrhotic patients with thrombocytopenia. Eur J Intern Med 2017;38:79-82.

15. Kalambokis GN, Oikonomou A, Christou L et al. von Willebrand factor and procoagulant imbalance predict outcome in patients with cirrhosis and thrombocytopenia. J Hepatol 2016;65:921-8.

16. Schulman S, Kearon C. Subcommittee on Control of Anticoagulation of the Scientific and Standardization Committee of the International Society on Thrombosis and Haemostasis. Definition of major bleeding in clinical investigations of antihemostatic medicinal products in non-surgical patients. J Thromb Haemost 2005;3:692-4.

17. Schulman S, Angerås U, Bergqvist $\mathrm{D}$ et al. Definition of major bleeding in clinical investigations of antihemostatic medicinal products in surgical patients. J Thromb Haemost 2010;8:202-4.

18. Violi F, Corazza GR, Caldwell SH et al. Portal vein thrombosis relevance on liver cirrhosis: Italian Venous Thrombotic Events Registry. Intern Emerg Med 2016;11:1059-66.

19. North Italian Endoscopic Club for the Study and Treatment of Esophageal Varices. Prediction of the first variceal hemorrhage in patients with cirrhosis of the liver and esophageal varices. A prospective multicenter study. N Engl J Med 1988;319:983-9.

20. Garcia-Tsao G, Sanyal AJ, Grace ND et al. Prevention and management of gastroesophageal varices and variceal hemorrhage in cirrhosis. Hepatology 2007;46:922-38.

21. Rodríguez-Castro KI, Antonello A, Ferrarese A. Spontaneous bleeding or thrombosis in cirrhosis: What should be feared the most? World J Hepatol 2015;7:1818-27.

22. D'Amico G, De Franchis R. Cooperative Study Group. Upper digestive bleeding in cirrhosis. Post-therapeutic outcome and prognostic indicators. Hepatology 2003;38:599-612.

23. Shah NL, Northup PG, Caldwell SH. A clinical survey of bleeding, thrombosis, and blood product use in decompensated cirrhosis patients. Ann Hepatol 2012;11:686-90.

24. Kalafateli M, Triantos CK, Nikolopoulou V et al. Non-variceal gastrointestinal bleeding in patients with liver cirrhosis: a review. Dig Dis Sci 2012;57:2743-54.

25. Huang H-H, Lin H-H, Shih Y-L et al. Spontaneous intracranial hemorrhage in cirrhotic patients. Clin Neurol Neurosurg 2008;110:253-8.

26. Lai CH, Cheng PY, Chen YY. Liver cirrhosis and risk of intracerebral hemorrhage: a 9-year follow-up study. Stroke 2011;42:2615-7.

27. Lisman T, Porte RJ. Rebalanced hemostasis in patients with liver disease: evidence and clinical consequences. Blood 2010;116:878-85.

28. Thakrar SV, Mallett SV. Thrombocytopenia in cirrhosis: impact of fibrinogen on bleeding risk. World J Hepatol 2017;9:318-25.

29. Desborough MJ, Kahan BC, Stanworth SJ et al. Fibrinogen as an independent predictor of mortality in decompensated cirrhosis and bleeding. Hepatology 2017;65:1079-80.

30. Ge PS, Runyon BA. Treatment of patients with cirrhosis. N Engl J Med 2016;375:767-77.

31. Burza MA, Marschall HU, Napoleone L et al. The 35-year odyssey of beta blockers in cirrhosis: any gender difference in sight? Pharmacol Res 2017;119:20-26.

32. D'Andrea G, Chetta M, Margaglione M. Inherited platelet disorders: thrombocytopenias and thrombocytopathies. Blood Transfus 2009;7:278-92.

33. McQuaid KR, Laine L. Systematic review and meta-analysis of adverse events of low-dose aspirin and clopidogrel in randomized controlled trials. Am J Med 2006;119:624-38.

34. Under the auspices of the Italian Association for the Study of Liver Diseases (AISF) and the Italian Society of Internal Medicine (SIMI). Hemostatic balance in patients with liver cirrhosis: report of a consensus conference. Dig Liver Dis 2016;48:455-67.

35. Violi F, Pignatelli P. Eltrombopag before procedures in patients with cirrhosis and thrombocytopenia. N Engl J Med 2012;367:2056. 